International Journal of Pure and Applied Mathematics

Volume 106 No. 3 2016, 851-857

ISSN: 1311-8080 (printed version); ISSN: 1314-3395 (on-line version)

url: http://www.ijpam.eu

doi: 10.12732/ijpam.v106i3.11

ijpam.eu

\title{
ON T-REPRESENTABILITY OF THE CYCLIC SUBGROUPS OF THE SYMMETRIC GROUP-I
}

\author{
P. Sini ${ }^{1}$, P.T. Ramachandran ${ }^{2}$ \\ ${ }^{1,2}$ Department of Mathematics \\ University of Calicut \\ Malappuram Dt., Pin: 673635, Kerala, INDIA
}

\begin{abstract}
In this paper we investigate the group of homeomorphisms of topological spaces. A subgroup $K$ of the group $S(X)$ of all permutations of a set $X$ is called $t$-representable on $X$ if there exists a topology $T$ on $X$ such that the group of homeomorphisms of $(X, T)=K$. We determine the $t$-representability of groups generated by a permutation which is a product of disjoint cycles having equal lengths.
\end{abstract}

AMS Subject Classification: 54H15, 20B35

Key Words: cyclic groups, group of homeomorphisms, permutation groups, t-representable

\section{Introduction}

Let $H(X, T)$ denote the group of all homeomorphisms of a topological space $(X, T)$ and $H(X, T)$ is a subgroup of the symmetric group $S(X)$. A subgroup $K$ of the group $S(X)$ of all permutations of a set $X$ is called $t$-representable on $X$ if there exists a topology $T$ on $X$ such that the group of homeomorphisms of $(X, T)=K$. The determination of $t$-representable subgroups of $S(X)$ is a very difficult and interesting problem. In [3], [4], P. T. Ramachandran showed that

Received: October 28, 2015

Published: February 27, 2016 (c) 2016 Academic Publications, Ltd.

url: www.acadpubl.eu 
no nontrivial proper normal subgroups of the group of all permutations of a set $X$ can be the group of homeomorphisms of $(X, T)$ for any topology $T$ on $X$. If $X=\left\{a_{1}, a_{2}, \ldots, a_{n}\right\}, \mathrm{n} \geq 3$, the group of permutations of $X$ generated by the cycle $\left(a_{1}, a_{2}, \ldots, a_{n}\right)$ cannot be represented as the group of homeomorphisms of $(X, T)$ for any topology $T$ on $X$ whereas if $X$ is an infinite set, then the cyclic group generated by an infinite cycle can be represented as the group of homeomorphisms of $(X, T)$ for a topology $T$ on $X$ see[3] and [5]).

In [6] it is proved that the direct sum of finite $t$-representable permutation groups is $t$-representable, every permutation group of order two is $t$ representable and also determined the $t$-representability of finite transitive permutation groups.

Basic terminology about topological spaces and groups will be consistent with [2], [7]. In this paper we continue the study carried out in [6].

\section{Main Results}

We investigate the $t$-representability of cyclic subgroups of $S(X)$ generated by a product of disjoint cycles having equal length.

We need the following theorem.

Theorem 1. [4] Let $X=\left\{a_{1}, a_{2}, \ldots a_{n}\right\}, n \geq 3$ and $H$ be the group of permutations on $X$ generated by the cycle $\left(a_{1}, a_{2}, \ldots a_{n}\right)$. Then $H$ is not t-representable on $X$.

Now we determine the $t$-representability of permutation groups generated by a permutation which is a product of two disjoint cycles that have equal length $n$ where $n \geq 3$.

Susan J. Andima and W. J Thron [1] associated with each topology $T$ on a set $X$ with a preorder relation ' $\leq$ ' on $X$ defined by $a \leq b$ if and only if every open set containing $b$ contains $a$. Then the group of homeomorphisms of $(X, T)$ onto itself is equal to the group of order isomorphisms of $(X, \leq)$, which is denoted by $G(X, \leq)$.

The next definition appears in [7].

Definition 2. $T_{0}$ Space: A topological space $(X, T)$ is said to be a $T_{0}$ space if given any two distinct points in $X$, there exist an open set which contains one of them but not the other.

So $(X, T)$ is a $T_{0}$ space if and only if $(X, \leq)$ is a partially ordered set. If $X$ is a finite nonempty set, then the partially ordered set $(X, \leq)$ has both maximal 
and minimal elements. Also an order isomorphism of $(X, \leq)$ maps maximal elements to maximal elements and minimal elements to minimal elements.

Theorem 3. If $\sigma$ is a permutation on $X$ which is a product of two disjoint cycles having equal length $n$ where $n \geq 3$, then the group generated by $\sigma$ is not t-representable on $X$.

Proof. Let $\sigma=\left(a_{1}, a_{2}, \ldots, a_{n}\right)\left(b_{1}, b_{2}, \ldots, b_{n}\right)$ and $K$ be the group generated by $\sigma$. Decompose $X$ as $X_{1} \cup X_{2}$ where $X_{1}=\left\{a_{1}, a_{2}, \ldots a_{n}\right\}$ and $X_{2}=\left\{b_{1}, b_{2}, \ldots b_{n}\right\}$. Assume that $K$ is $t$-representable on $X$. Then there exist a topology $T$ on $X$ such that the group of homeomorphisms on $X$, $H(X, T)=K$. Since there exist no transposition on $X$ as homeomorphism of $(X, T)$, the space must be $T_{0}$. Let $(X, \leq)$ be the corresponding partially ordered set. Since $X$ is a finite set, $(X, \leq)$ has both maximal and minimal elements. If an element is both minimal and maximal, then all the elements are minimal and maximal and hence $H(X, T)=S(X)$, which is not possible. So a minimal element can not be a maximal element. Then either $X_{1}$ or $X_{2}$ is the set of all minimal elements.

Assume that $X_{1}$ is the set of all minimal elements. Then the elements of $X_{2}$ is the set of all maximal elements. Thus the elements of $X_{i}$ are incomparable to each other for $i=1$, 2. So there exist atleast one $a_{j} \in X_{1}$ and $b_{i} \in X_{2}$ such that $b_{i}$ succeeds $a_{j}$. Otherwise $G(X, \leq)=S(X)$, which is a contradiction. Since $G(X, \leq)=K$, if $b_{i}$ succeeds $a_{j}$, then $\sigma^{h}\left(b_{i}\right)$ succeeds $\sigma^{h}\left(a_{j}\right)$ for $h=$ $1,2, \ldots, n-1$ and this gives $a_{j \oplus(h-1)}<b_{i \oplus(h-1)}$ where $\oplus$ denotes addition modulo $n$. So without loss of generality we can assume that $b_{1}$ atleast succeeds $a_{1}$. If $b_{1}$ succeeds only $a_{1}$, then $\left(a_{1}, a_{i}\right)\left(b_{1}, b_{i}\right)$, where $i=2,3, \ldots n$ are order isomorphisms, which is not possible. Now assume that $b_{1}$ succeed $k$ elements in $X_{1}$ namely $a_{1}, a_{2}, \ldots, a_{k}$ where $1<k \leq n$. Then each $b_{i} \in X_{2}$ exactly succeed $k$ elements in $X_{1}$ namely $a_{i}, a_{i \oplus 1}, \ldots, a_{i \oplus(k-1)}$. Then

$$
\mathscr{B}=\left\{\left\{a_{i}\right\},\left\{a_{i}, a_{i \oplus 1}, \ldots, a_{i \oplus k-1}, b_{i}\right\}, i=1,2, \ldots, n\right\}
$$

form a base for $T$.

If $k=n$, then $H(X, T)=S\left(X_{1}\right) \oplus S\left(X_{2}\right)$, which is not possible. So assume that $1<k<n$

For $j=1,2, \ldots n$, define $h: X \rightarrow X$ as

$$
h(x)= \begin{cases}a_{j \oplus n-(i-1)} & \text { if } x=a_{i}, 1 \leq i \leq n \\ b_{j \oplus n-(k+i-2)} & \text { if } x=b_{i}, 1 \leq i \leq n .\end{cases}
$$

Now we claim that $h$ is a is a homeomorphism on $(X, T)$ onto itself. For let $U \in \mathscr{B}$. Then either $U$ is a singleton or $U=\left\{a_{l}, a_{l \oplus 1}, \ldots, a_{l \oplus(k-1)}, b_{l}\right\}$, where 
$1 \leq l \leq n$. If $U$ is a singleton, then $h(U) \in \mathscr{B}$. Now let

$$
U=\left\{a_{l}, a_{l \oplus 1}, \ldots, a_{l \oplus(k-1)}, b_{l}\right\} .
$$

Then

$$
\begin{aligned}
h(U)= & \left\{a_{j \oplus n-(l-1)}, a_{j \oplus n-l}, a_{j \oplus n-(l+1)}, \ldots,\right. \\
& \left.a_{j \oplus n-(l+k-2)}, b_{j \oplus n-(l+k-2)}\right\} \\
= & \left\{a_{j \oplus(n-l)+1)}, a_{j \oplus n-l}, a_{j \oplus(n-l)-1)}, \ldots,\right. \\
& \left.a_{j \oplus(n-(l+k)+2)}, b_{j \oplus(n-(l+k)+2)}\right\} \\
= & \left\{a_{j \oplus n-(l+k)+2}, a_{j \oplus n-(l+k)+3}, \ldots,\right. \\
& \left.a_{j \oplus n-(l+k)+k}, a_{j \oplus n-(l+k)+(k+1)}, b_{j \oplus n-(l+k)+2)}\right\} \\
= & \left\{a_{p}, a_{p \oplus 1}, a_{p \oplus 2}, \ldots a_{p \oplus(k-1)}, b_{p}\right\}, \\
& \text { where } p=j \oplus n-(l+k)+2 .
\end{aligned}
$$

Thus $h(U) \in \mathscr{B}$. Observe that $h$ is the product of transpositions and hence $h^{-1}=h$. Consequently, we have $h^{-1}(U) \in \mathscr{B}$. This is true for all $U \in \mathscr{B}$. So $h$ is a homeomorphism on $(X, T)$, which is a contradiction to the fact that $H(X, T)=K$. This completes the proof.

Example 4. Let $X=\left\{a_{1}, a_{2}, a_{3}, b_{1}, b_{2}, b_{3}\right\}$ and

$$
\sigma=\left(a_{1}, a_{2}, a_{3}\right)\left(b_{1}, b_{2}, b_{3}\right)
$$

Then the cyclic group generated by $\sigma$ is,

$$
<\sigma>=\left\{I,\left(a_{1}, a_{2}, a_{3}\right)\left(b_{1}, b_{2}, b_{3}\right),\left(a_{1}, a_{3}, a_{2}\right)\left(b_{1}, b_{3}, b_{2}\right)\right\} .
$$

Suppose $\langle\sigma\rangle$ is $t$-representable on $X$, then there exist a topology $T$ on $X$ such that $H(X, T)=\langle\sigma\rangle$. Consider $(X, \leq)$, then arguing as in the Theorem 3 either $X_{1}=\left\{a_{1}, a_{2}, a_{3}\right\}$ or $X_{2}=\left\{b_{1}, b_{2}, b_{3}\right\}$ is the set of all minimal elements. Without loss of generality we can assume that $X_{1}$ is the set of all minimal elements. So the possible partial orders on $X$ are essentially as in the following figure 


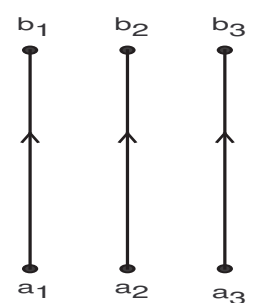

t

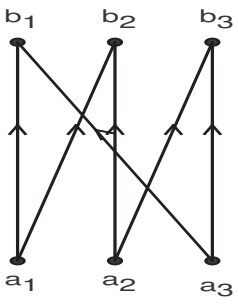

$\mathbf{u}$

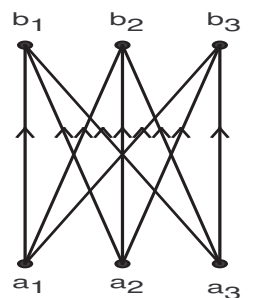

V
$\left(X, \leq_{1}\right)$
$\left(X, \leq_{2}\right)$
$\left(X, \leq_{3}\right)$

Then

$$
\begin{aligned}
G\left(X, \leq_{1}\right)= & \left\{I,\left(a_{1}, a_{2}\right)\left(b_{1}, b_{2}\right),\left(a_{1}, a_{3}\right)\left(b_{1}, b_{3}\right),\left(a_{2}, a_{3}\right)\left(b_{2}, b_{3}\right),\right. \\
& \left.\left(a_{1}, a_{2}, a_{3}\right)\left(b_{1}, b_{2}, b_{3}\right),\left(a_{1}, a_{3}, a_{2}\right)\left(b_{1}, b_{3}, b_{2}\right)\right\}, \\
G\left(X, \leq_{2}\right)= & \left\{I,\left(a_{1}, a_{2}\right)\left(b_{1}, b_{3}\right),\left(a_{1}, a_{3}\right)\left(b_{2}, b_{3}\right),\left(a_{2}, a_{3}\right)\left(b_{1}, b_{2}\right),\right. \\
& \left.\left(a_{1}, a_{2}, a_{3}\right)\left(b_{1}, b_{2}, b_{3}\right),\left(a_{1}, a_{3}, a_{2}\right)\left(b_{1}, b_{3}, b_{2}\right)\right\}, \\
\text { and } G\left(X, \leq_{3}\right)= & S\left(X_{1}\right) \oplus S\left(X_{2}\right) .
\end{aligned}
$$

So there exist no partial orders on $X$ such that the group of order isomorphisms is the group generated $\sigma$. Hence $\langle\sigma\rangle$ is not $t$-representable on $X$.

Now we consider the $t$-representability of the cyclic group generated by a permutation on $X$ which is an arbitrary product of more than two disjoint cycles having equal lengths $n$ where $n>2$. Here we prove that such cyclic subgroups of symmetric group $S(X)$ are $t$-representable on $X$.

Theorem 5. If $\sigma$ is a permutation on $X$ which is an arbitrary product of more than two disjoint cycles having equal length $n$ where $n>2$, then the group generated by $\sigma,\langle\sigma\rangle$ is t-representable on $X$.

Proof. Let

$$
\sigma=\prod_{i \in I} C_{i}
$$


where $\left\{C_{i}, i \in I\right\}$ be an indexed family of disjoint cycles having equal length $n$ where $n>2$ and $|I|>2$. Let $C_{i}=\left(a_{i 1}, a_{i 2}, \ldots, a_{i n}\right)$ for every $i \in I$ and $X=\cup_{i \in I} X_{i}$.

By assuming axiom of choice, well-order the set $I$ by the order relation $<$. Let $i_{0}$ be the first element of $I$. Now consider the set $\left\{i \in I: i_{0}<i\right\}$ and $i_{1}$ denote the first element of the set $I \backslash\left\{i_{0}\right\}$. In general $i_{j}$ denote the first element of the set $I \backslash\left\{i_{0}, i_{1}, i_{2}, \ldots, i_{j-1}\right\}$.

Define a base $\mathscr{B}$ by

$$
\mathscr{B}=\left\{A_{i j}: i \in I, j=1,2, \ldots, n\right\}
$$

where

$$
A_{i j}= \begin{cases}\left\{a_{k j}: k \leq i\right\} & \text { if } i<i_{2} \\ \left\{a_{k j: k \leq i}\right\} \cup\left\{a_{i_{0}} \oplus \oplus(n-1)\right. & \text { if } i \geq i_{2} .\end{cases}
$$

and $T$ be the topology on $X$ having base $\mathscr{B}$. It can be verified that $\sigma$ is a homeomorphism of $(X, T)$. Then all powers of $\sigma$ are homeomorphisms of $(X, T)$. Hence $\langle\sigma\rangle \subseteq H(X, T)$

Conversely let $h \in H(X, T)$. We have to prove that $h$ is of the form $\sigma^{m}$ for some $m$ such that $1 \leq m \leq n$. Since $h$ is a homeomorphism, $h\left(A_{i_{0} 1}\right)=A_{i_{0} m}$ for some $m, 1 \leq m \leq n$. Thus $h\left(a_{i_{0} 1}\right)=a_{i_{0} m}$. If $h\left(a_{i_{0} 1}\right)=a_{i_{0} m}$, then $h\left(a_{i 1}\right)=a_{i m}$ for all $i \in I$ and $h\left(a_{i_{0} n}\right)=a_{i_{0} m \oplus(n-1)}$. Now let $h\left(a_{i_{0} 2}\right)=a_{i_{0} p}$. Then $h\left(a_{i 2}\right)=a_{i p}$ for all $i \in I$ and $h\left(a_{i_{0} 2 \oplus(n-1)}\right)=a_{i_{0} p \oplus(n-1)}$. That is $h\left(a_{i_{0} 1}\right)=a_{i_{0} p \oplus(n-1)}$. By the fact that $h\left(a_{i_{0} 1}\right)=a_{i_{0} m}$ we have $m=p \oplus(n-1)$. This implies that $p=m \oplus 1$.

Now assume that $h\left(a_{i_{0} \alpha}\right)=a_{i_{0} \beta}$ for some $\alpha$ and $\beta$, thus $h\left(a_{i \alpha}\right)=a_{i \beta}$ for all $i \in I$ and $h\left(a_{i_{0} \alpha \oplus(n-1)}\right)=a_{i_{0} \beta \oplus(n-1)}$. Now let $h\left(a_{i_{0} \alpha \oplus 1}\right)=a_{i_{0} \gamma}$. Then $h\left(a_{i_{0} \alpha \oplus 1 \oplus(n-1)}\right)=a_{i_{0} \gamma \oplus(n-1)}$, which implies $h\left(a_{i_{0} \alpha}\right)=a_{i_{0} \gamma \oplus(n-1)}$ and we obtain that $\beta=\gamma \oplus(n-1)$ and so $\gamma=\beta \oplus 1$. Consequently we get $h\left(a_{i \alpha \oplus 1}\right)=a_{i \beta \oplus 1}$ for all $i \in I$ and so $h\left(a_{i k}\right)=a_{i k \oplus(m-1)}$ for all $i \in I$ and $1 \leq k \leq n$. Hence $h\left(a_{i k}\right)=\sigma^{m}\left(a_{i k}\right)$ for all $i \in I$ and $1 \leq k \leq n$, which implies that $h=\sigma^{m}$. Thus $h \in\langle\sigma\rangle$. So $\left.H(X, T) \subseteq<\sigma\right\rangle$. This completes the proof.

Theorem 6. Let $X$ be any set and $H$ be the group on $X$ generated by $\sigma=\prod_{i \in I} C_{i}$ where where $\left\{C_{i}, i \in I\right\}$ be an indexed family of disjoint cycles having equal length $n$. Then

1. $H$ is $t$-representable if $|I|>2$ or $n<3$.

2. $H$ is not $t$-representable if $|I| \leq 2$ and $n \geq 3$. 
Proof. In [6], it is proved that every permutation group of order two is $t$ representable. So if the length of each cycle of the permutation $\sigma$ is less than three, then $\langle\sigma\rangle$ is $t$-representable. Thus $H$ is $t$-representable if $n<3$. Now $H$ is $t$-representable if $I>2$ by Theorem 5 and $H$ is not $t$-representable if $|I| \leq 2$ and $n \geq 3$ by Theorems 1 and 3 .

\section{Conclusion}

It is convenient to point out that we determined the $t$-representability of groups generated by a permutation which is a product of disjoint cycles having equal lengths.

\section{Acknowledgements}

The first author acknowledges the financial support from University Grants Commission, Govt. of India for awarding the teacher fellowship for doing the research and expresses sincere thanks to Dr. Ramachandran P. T, Associate Professor, Department of Mathematics, University of Calicut for his guidance and critical suggestions through out the preparation of this paper.

\section{References}

[1] Andima S. J. and Thron W. J., Order Induced Topological Properties, Pacific Journal of Mathematics, Vol-75, No. 2, (1978), 297-318.

[2] Dixon J. D. and Mortimer B. , Permutation Groups, Graduate Text in Mathematics, Springer-Verlag, New York, (1996).

[3] Ramachandran P.T , Groups of Homeomorphisms and Normal Subgroups of the Group of Permutations, Internat. J. Math. Math. Sci. Vol-14, No.3, (1991), 475-480.

[4] Ramachandran P.T., Some Problems in Set Topology Relating Group of Homeomorphisms and Order, Thesis for Ph. D. Degree, CUSAT (1985).

[5] Ramachandran P.T , The Group of Homeomorphisms and the L-fuzzy Gomeomorphisms, Proceedings of the National Conference on Mathematical Modelling, Kottayam, (2002).

[6] Sini. P. and Ramachandran P.T., On Group of Homeomorphisms, Bulletin of KMA, (2012), 55-63.

[7] Willard S., General Topology, Dover Publications, INC. New York, (2004). 
